\title{
Sexual activity and the risk of prostate cancer: Review article
}

\author{
Ahmed Fouad Kotb, Ahmad Beltagy, Asmaa Mohamed Ismail, Mohamed Mohie Hashad \\ Urology Department, Faculty of Medicine, Alexandria University, Alexandria, Egypt.
}

\begin{abstract}
Summary Introduction: Sexual activity can affect prostate cancer pathogenesis in a variety of ways; including the proposed high androgen status, risk of sexually transmitted infections and the potential effect of retained carcinogens within the prostatic cells.

Methods: PubMed review of all publications concerning sexual activity and the risk of prostate cancer was done by two researchers.

Results: Few publications could be detected and data were classified as a prostate cancer risk in association with either heterosexual or homosexual activities.

Conclusion: Frequent ejaculation seems to be protective from the development of prostate cancer. Multiple sexual partners may be protective from prostate cancer, excluding the risk of sexually transmitted infections. Homosexual men are at a greater risk for the diagnosis of prostate cancer.
\end{abstract}

KEY WORDS: Prostate cancer; Ejaculation; Homosexuality; Heterosexuality.

Submitted 16 January 2015; Accepted 31 March 2015

Sexual activity is hypothesized to affect prostate cancer (PCa) pathogenesis through numerous etiologic pathways. One of the proposed mechanism associates increased sexual activity to higher androgenic activity that may be an indicator for a higher PCa risk (1).

Another mechanism proposes that sexual activity increase exposure to sexually transmitted infectious diseases (STDs), which have been hypothesized to play a role in PCa development (2). A different hypothesis suggests that a reduced ejaculation frequency in otherwise normal men might be an etiologic risk factor for PCa. Such proposition is based on the theory that infrequent ejaculation causes carcinogenic secretions to be retained within the prostatic acini (3).

\section{Heterosexuality and PCa}

Several studies were found concerning the heterosexual activity and prostate cancer risk. Rosenblatt et al. (4) suggested that there is a direct positive correlation between the number of lifetime female sexual partners and the risk of prostate cancer in middle-aged men.

The increased risk associated with having more than one female sexual partner was observed during most age periods, and was associated with increased exposure to sexually transmitted diseases. A meta-analysis in 2002 confirmed the positive association between multiple female sexual partners,

STDs and the resulting higher risk of prostate cancer (RR 1.2) (5) Fernández et al. (6) found that men who had sexual intercourse more than 7 times per week had a significant positive risk of having prostate cancer. Included men were in the old age group, with only $7 \%$ of men younger than 55 years, and that was associated with the risk of having STDs.

Giles et al. (7) studied 2.338 men and found no association of prostate cancer with the number of sexual partners and argued against infection as a cause of prostate cancer. They, however, could detect that men who averaged 5 or more ejaculations weekly in their 20 s had a relative risk of 0.66 compared to those who ejaculated less frequently.

Spence et al. (8) reported a protective effect of having several female sexual partners over the lifetime. In their study, they found that those men having $>20$ female sexual partners had a reduced risk of overall (OR 0.72) and less aggressive PCa (OR 0.68) compared to men reporting having had only one female sexual partner over the lifetime. They explained this protective effect may be related to higher ejaculation frequency which is supposed to be PCa protective.

Frequent ejaculation is thought to reduce the concentration of carcinogenic substances within prostatic fluid (3) or reduce production of intraluminal prostatic crystalloids (9).

Early on 1986, a study found that the average ejaculatory frequency in PCa patients was significantly lower than in the control group and mentioned that reduced ejaculatory frequency appears to promote the pathogenesis of PCa by retained prostatic secretions promoting dysplasia of the prostatic gland epithelium (10).

Age of the first intercourse was a factor that was also studied in the literatures. Ahmadi et al. (11) found that early age of first marriage was significantly associated with a lower risk of prostate cancer. A study in 1993 detected that PCa patients were significantly older at the time of their first marriage, compared with men who first married under the age of 25 years (12). 
In 2004, a large prospective study on 29.342 men by Leitzmann et al. (13) concluded that higher ejaculation frequency was related to decreased risk of total and organ confined prostate cancer.

The relative risk for men reporting 21 or more ejaculations per month across a lifetime was 0.67 (33\% lower risk) compared to 0.89 for men reporting 4 to 7 ejaculations per month across a lifetime. Chavez et al. (14) conducted a retrospective study on 4,974 men with erectile dysfunction (ED).

They could find that men treated for ED with phosphodiesterase inhibitors (47.5\%) had a significantly lower risk for prostate cancer than non-treated patients that could be attributed to more frequent ejaculations and retaining of sexual activities.

\section{Homosexuality AND PCA}

Sexual orientation and $\mathrm{PCa}$ has received little attention in the literature. A published study in 2002 found no association between sexual orientation and PCa. (4) Potential mechanisms underlying a possible greater risk of $\mathrm{PCa}$ among men having had several male partners or bisexuals are still unclear. Homosexual men have been found to report more often a diagnosis of prostate cancer than heterosexual men (15). HIV is more in homosexual men and being immunosuppressed, making them more vulnerable to develop cancer (15).

Another potential mechanism attributes physical trauma to the prostate. Receptive anal intercourse, significantly result in increased serum PSA levels causing higher PCa diagnosis rates (16).

Potentially, the physical pounding of the prostate gland itself may possibly lead to a greater risk of PCa like previous studies have linked the receipt of physical trauma to with breast and testicular cancers.

Spence et al. (8) found that men who ever had 2-3 male sexual partners over their lifetime were at a significantly greater risk of less aggressive PCa compared to men who never had male partners (OR 3.01).

\section{Conclusion}

Frequent ejaculation seems to be a protective factor for the development of prostate cancer. Homosexual men may be at a higher risk of being diagnosed with prostate cancer, during their lifetime, more than heterosexual men.

\section{REFERENCES}

1. Krain LS. Some epidemiologic variables in prostatic carcinoma in California. Prev Med. 1974; 3:154- 9.

2. Taylor ML, Mainous AG 3rd, Wells BJ. Prostate cancer and sexually transmitted diseases: a meta-analysis. Fam Med. 2005; 37:506-12.

3. Isaacs JT. Prostatic structure and function in relation to the etiology of prostatic cancer. Prostate. 1983; 4:351-66.

4. Rosenblatt KA, Wicklund KG, Stanford JL. Sexual factors and the risk of prostate cancer. Am J Epidemiol. 2001; 153:1152-8.

5. Dennis LK, Dawson DV. Meta-analysis of measures of sexual activity and prostate cancer. Epidemiology. 2002; 13:72-9.

6. Fernández L, Galán Y, Jiménez R, et al. Sexual behaviour, history of sexually transmitted diseases, and the risk of prostate cancer: a case-control study in Cuba. Int J Epidemiol. 2005; 34:193-7.

7. Giles GG, Severi G, English DR, et al. Sexual factors and prostate cancer. BJU Int. 2003; 92:211-6.

8. Spence AR, Rousseau MC, Parent MÉ. Sexual partners, sexually transmitted infections, and prostate cancer risk. Cancer Epidemiol. $2014 ; 38: 700-7$.

9. Del Rosario AD, Bui HX, Abdulla M, Ross JS. Sulfur-rich prostatic intraluminal crystalloids: a surgical pathologic and electron probe x-ray microanalytic study. Hum Pathol. 1993; 24:1159-67.

10. Banerjee AK. Carcinoma of prostate and sexual activity. Urology. 1986; 28:159.

11. Ahmadi H, Allameh F, Baradaran N, et al. Circulating sex hormones play no role in the association between sexual activity and the risk of prostate cancer. J Sex Med. 2011; 8:905-13.

12. La Vecchia C, Franceschi S, Talamini R, et al. Marital status, indicators of sexual activity and prostatic cancer. J Epidemiol Community Health. 1993; 47:450- 3.

13. Leitzmann MF, Platz EA, Stampfer MJ, Willett WC, Giovannucci E. Ejaculation frequency and subsequent risk of prostate cancer. JAMA. 2004; 291:1578- 86.

14. Chavez AH, Coffield KS, Rajab MH, Jo C. Incidence rate of prostate cancer in men treated for erectile dysfunction with phosphodiesterase type 5 inhibitors: retrospective analysis. Asian J Androl 2013; 15:246-248.

15. Boehmer U, Miao X, Ozonoff A. Cancer survivorship and sexual orientation. Cancer. 2011; 117:3796-804.

16. Stephan C, Jung K, Diamandis EP, et al. Prostate-specific antigen, its molecular forms, and other kallikrein markers for detection of prostate cancer. Urology. 2002; 59:2-8.

\author{
Correspondence \\ Ahmed Fouad Kotb; M.D, PhD, MRCS, FEBU \\ drahmedfali@gmail.com \\ Ahmad Beltagy, MD \\ bil_doctor@hotmail.com \\ Asmaa Mohamed Ismail, MD \\ asmaaismail@rocketmail.com \\ Urology Department, Faculty of Medicine, Alexandria University, \\ Azarita, Sultan Hussein Street, Alexandria, Egypt
}

